\title{
Algas verdes y euglénidos de la Puna argentina
}

\section{Green Algae and Euglenids of Argentinean Puna}

\author{
Mirande, Virginia1*; Beatriz C. Tracanna ${ }^{2-3}$ \\ 1 Instituto de Ficología, Fundación Miguel Lillo, Miguel Lillo 251, (4000) San Miguel de Tucumán, Ar- \\ gentina. \\ a Facultad de Ciencias Naturales e Instituto Miguel Lillo. Universidad Nacional de Tucumán. Miguel Lillo \\ 205, (4000) San Miguel de Tucumán, Argentina. \\ 3 CONICET. \\ * Autor corresponsal: virginiamirande@yahoo.com.ar
}

Resumen - Mirande, Virginia; Beatriz C. Tracanna. 2017. "Algas verdes y euglénidos de la puna argentina". Lilloa 54 (2). El objetivo de este trabajo fue contribuir al conocimiento cualitativo de las clorofitas y euglenofitas de la puna argentina. Estos ambientes se caracterizan por ser sistemas extremos, dinámicos y frágiles, esenciales para el funcionamiento de pequeñas cuencas hidrográficas altoandinas. Durante el verano de 2005 se efectuó un muestreo que abarcó veintiséis cuerpos lénticos en las provincias de Jujuy, Salta y Catamarca. Las muestras se obtuvieron por el concentrado de 25 litros de agua a través de una red de plancton de $20 \mu \mathrm{m}$ de poro y fueron fijadas in situ con formaldehído $4 \%$. Las determinaciones algales se realizaron en laboratorio bajo microscopio binocular con dispositivo para dibujo. Se reconocieron 28 taxones que pertenecieron 24 a Chlorophyta y 4 a Euglenophyta. El número más alto de especies fue ocho en Los Enamorados [Jujuy] y sin registro en el $26 \%$ de los cuerpos de agua muestreados. De acuerdo a los resultados, hubo un predominio de especies raras, la mayoría cosmopolitas y de medios salinos. De ahí que diecisiete de las veintiocho entidades determinadas, es decir el 60,7\%, fueron encontradas en ambientes salinos. Chlamydomonas rubrifilum, Oedogonium sp. 1 y Stigeoclonium sp. 2 fueron halladas sólo en aguas hipersalinas, Chlamydomonas tremulans, Dedogonium sp. 3, Spirogyra sp. 1, Spirogyra sp. 2, Ulothrix pseudoflacca var. salina, Euglena ehrenbergii y Euglena proxima también estuvieron a salinidades menores. En el caso de Raciborskiella salina fue detectada en aguas tanto salinas como salinas-hipersalinas con conductividades de hasta $22500 \mu \mathrm{S} / \mathrm{cm}$. Los taxones de algas verdes y euglénidos enunciados en esta publicación son nuevas citas para estos humedales debido a que no se contaba con antecedentes previos para estos grupos. Por otro lado, Ulothrix pseudoflacca var. salina es mencionada por primera vez para el Noroeste Argentino (NOA).

Palabras clave: Clorofitas, euglenofitas, puna, NOA.

Abstract - Mirande, Virginia; Beatriz C. Tracanna. 2017. "Green Algae and Euglenids of Argentinean Puna". Lilloa 54 (2). The aim of this paper was to contribute to the qualitative knowledge of chlorophytes and euglenophytes of argentinean puna. These environments are extrem, dynamic and weak systems, essential for functionement of small andean watersheds. Sampling was realized during the summer of 2005 and this included twenty six lentic bodies corresponding to the provinces of Jujuy, Salta and Catamarca. Qualitative samples were obtained by the concentrate 25 liters of water through plankton net of $20 \mu \mathrm{m}$ and were fixed in situ with formaldehyde $4 \%$. Algal determinations were performed under binocular microscope with drawing camera in laboratory. We recognized 28 taxa belonging to Chlorophyta (24) and Euglenophyta (4). The highest number of species was 8 in Los Enamorados [Jujuy) and without registration in $26 \%$ of water bodies sampled. According to the results, there was a predominance of rare species, the most cosmopolitan and saline environments. Hence seventeen of the twenty eight recognized entities (60.7\%) were in saline environments. Chlamydomonas rubrifilum, Dedogonium sp. 1 and Stigeoclonium sp. 2 were the ones found only in hypersaline 
waters while Chlamydomonas tremulans, Dedogonium sp. 3, Spirogyra sp. 1, Spirogyra sp. 2. Ulothrix pseudoflacca var. salina, Euglena ehrenbergii were also found at lower salinities. In the case of Raciborskiella salina it was detected in both salt and salt-hypersaline waters, with conductivities up to $22500 \mu \mathrm{S} / \mathrm{cm}$. The green algae and euglenids taxa statements in this publication are new appointments for these wetlands because there were not register about them. Furthermore, Ulothrix pseudoflacca var. salina is mentioned for the first time to the Northwest of Argentina (NWA).

Keywords: Chlorophytes, euglenophytes, puna, NWA.

\section{INTRODUCCIÓN}

Argentina registra una importante diversidad y abundancia de humedales, este término incluye a marismas, pantanos, turberas o superficies cubiertas de aguas estancadas o fluyentes, permanentes o temporales, naturales o artificiales, dulces, saladas o salobres e inclusive las extensiones de agua marina cuya profundidad en bajamar no exceda los seis metros (Canevari et al., 1998). Hasta el presente fueron reconocidos veintiún humedales como sitios Ramsar (Vecinos del Humedal, 2013), los cuales son valorados como: 1) reservas de agua, 2) recarga de acuíferos, 3) mitigación de erosiones e inundaciones, 4) reciclado de la materia orgánica, 5) retención, transformación y remoción de sedimentos, nutrientes y contaminantes, 6) refugio para otras especies, algunas seriamente amenazadas, 7) usos tradicionales, 8) otros. La palabra ramsar con la que se designa a los ambientes protegidos por leyes internacionales hace referencia a la ciudad iraní donde se realizó la Primera Convención sobre Humedales. El propósito principal de esta convocatoria llevada a cabo en 1971 fue la conservación y uso racional de los ecosistemas utilizados por las aves acuáticas para su nidificación (Coconier, 2005; Dirección de Recursos Ictícolas y Acuícolas, 2006).

Las escasas informaciones sobre estos recursos de fundamental importancia socioeconómica y ecológica están asociadas con las complejidades latitudinales y longitudinales que deben superarse para el estudio de los ecosistemas de altura en el Noroeste Argentino. Entre los antecedentes disponibles pueden mencionarse trabajos limnológicos (Halloy, 1978, 1982), planctónicos (Locascio de Mitrovich, 1986; Locascio de Mitrovich y Ceraolo, 1999; Locascio de Mitrovich et al.,
2005; Paggi y Villagra de Gamundi, 1980; Villagra de Gamundi, 1994, 1998; Maidana y Seeligmann, 2006; Mirande y Tracanna, 2007, 2009, 2015; Seeligmann y Maidana, 2003; Seeligmann et al., 2008) y de aves (Caziani y Derlindati, 1999, 2000; Caziani et al., 2001). Este estudio se realizó con el objetivo de contribuir al conocimiento cualitativo de las clorofitas y euglenofitas de la puna argentina.

En nuestro país la provincia geológica de la puna corresponde al límite austral del altiplano boliviano-peruano y se caracteriza por ser una altiplanicie escalonada entre numerosos volcanes que constituyen las máximas elevaciones de la cordillera de Los Andes (Caziani y Derlindati, 1999), estos conos volcánicos alcanzan grandes alturas como el volcán de Antofalla de 6100 msnm. En Catamarca termina en la cordillera de San Buenaventura, al norte del paso de San Francisco y la altura media de esta formación oscila entre $3600-3800 \mathrm{msnm}$. No se trata de una meseta porque presenta una serie de elevaciones o cordones con orientación general norte-sur, entre los cuales se desarrollan depresiones que contienen lagunas o salares de diversas dimensiones (por ejemplo: las lagunas Baya, del Salitre, Grande y los salares de Antofalla, Ratones, etc.). En relación a su basamento es cristalino con predominio de rocas metamórficas y efusivas paleozoicas a terciarias (Morlans, 1995). Este relieve puneño es consecuencia de la fracturación en bloques ocasionada por movimientos compresivos y de ascenso de la orogenia andina (Terciario y Cuaternario inferior) y de un drenaje centrípeto hacia zonas más bajas formando lagunas, vegas y salares. La mayoría de los cursos de agua son de regímenes temporarios debido 
a las características pluviométricas (lluvias predominantemente estivales), con valores anuales inferiores a $300 \mathrm{~mm}$ hacia el margen este más húmedo y en general menores a $200 \mathrm{~mm}$ en el resto de la puna. Las escasas precipitaciones hacen que la recarga de los acuíferos subterráneos sea baja, en esto último son fundamentales los procesos de hielo-deshielo en las altas cumbres debido a que la infiltración supera la evaporación. Los aportes dados por las granizadas esporádicas y el derretimiento de las nieves anuales son de menor valía. Los cursos temporarios de escaso caudal a la brevedad se infiltran dando lugar en algunos casos a la formación de vegas, mientras que los de volúmenes importantes arrastran grandes cargas de sedimentos que se depositan en amplios conos de deyección, rellenando valles y a veces se transforman en coladas de barro que generan en su base lagunas temporales o permanentes. Estas últimas pueden ser de agua dulce o salada, lo cual depende de las litologías atravesadas por sus tributarios y, por ende, las concentraciones de sales recibidas (INTA-UNSa, 2009).

Los ambientes acuáticos puneños se caracterizan por ser sistemas extremos, dinámicos y frágiles, esenciales para el funcionamiento de cuencas hidrográficas menores. Asimismo, proporcionan refugios temporales a aves migratorias como los flamencos andino, chilensis y de james y diversos mamíferos (burros, llamas, vicuñas, guanacos, chinchilla, entre otros), además de albergar especies de plantas y animales endémicas, es decir, exclusivas de estos ecosistemas. Las condiciones de fragilidad y extremidad están asociadas a causas naturales como la presencia de varios meses de sequías, altas irradiaciones y vientos intensos, amplitudes térmicas extremas y a actividades antrópicas tales como agricultura no sostenible, sobrepastoreo, minería a cielo abierto, entre otras (Caziani y Derlindati, 1999). En base a la morfología y características fisicoquímicas las lagunas de la puna fueron clasificadas como salinas, salinas-hipersalinas e hipersalinas (Caziani y Derlindati, 1999, 2000), lo cual fue coincidente con el criterio de salobres e hiper- salobres dado por Fjeldsa (1985). En líneas generales las de baja salinidad son pequeñas, profundas y con macrófitas, mientras que las hipersalinas son de grandes dimensiones y someras, ubicándose entre ambas las salinashipersalinas caracterizadas por sus fluctuaciones en sales (Caziani y Derlindati, 2000; Mirande y Tracanna, 2015).

En Jujuy se destacan dos sitios Ramsar, el sistema de Vilama (220 $30^{\prime} \mathrm{S}, 6^{\circ} 6^{\circ} 5^{\prime} \mathrm{W}$ ) y Pozuelos ( $22^{\circ} 20^{\prime} \mathrm{S} 66^{\circ} 00^{\prime} \mathrm{W}$ ). El primero abarca una superficie de 157000 ha que contiene más de una docena de lagunas alimentadas por aguas surgentes o de deshielo. El segundo denominado Monumento Natural Laguna de Pozuelos comprende un área de 16470 ha, la cual incluye esta laguna que es la de mayor extensión en la puna jujeña; no obstante, su cubeta ha disminuido notablemente en los últimos años, alcanzando una superficie máxima de $110 \mathrm{~km}^{2} \mathrm{y}$, por consiguiente, su salinidad es muy variable (Caziani y Derlindati, 2000; Caziani et al., 2001; Coconier, 2005). En Catamarca se protegieron a nivel provincial grandes extensiones, además de ciertas reservas privadas de menores dimensiones, algunas con serios problemas provenientes de una creciente urbanización que conlleva a un incremento del turismo y de la minería, del sobrepastoreo por una ganadería intensiva (llamas, ovejas y cabras), entre otros impactos ambientales (pesca deportiva, riego y consumo humano en tributarios, etc.). Ejemplo, laguna Blanca (somera y salina) conocida desde 1979 como la Reserva Natural de Vida Silvestre Laguna Blanca (primera unidad catamarqueña protegida) presenta en sus cercanías pequeños asentamientos como Laguna Blanca y Corral Blanco. La ubicación de esta reserva está indicada a través de carteles aunque no se efectúan actividades de control y vigilancia (Sureda et al., 2005). Las lagunas Grande (somera y salina, congelada en invierno), Purulla (hipersalina, sobre un pedimento minero) y La Alumbrera (profunda y salina, con macrófitas, refugio invernal de aves acuáticas) fueron incluidas dentro del ítem Monumento Natural y Reserva Provincial de Uso Múltiple presentado en el Proyecto de 
Áreas Protegidas Las Parinas de la Administración de Parques Nacionales. En relación con este último cuerpo lacustre es importante destacar la existencia de la pujante población de Antofagasta de la Sierra y sus consecuentes problemas antrópicos (Di Giacomo y Coconier, 2005; Sureda y Caziani, 2007 a, b, c).

\section{MATERIALES Y MÉTODOS}

Con referencia a los puntos escogidos para este estudio, algunos protegidos a escalas internacional, nacional o provincial, son coincidentes con los expuestos en Mirande y Tracanna (2015). Aparte de la información aportada en esta publicación, mayores de- talles sobre las zonas seleccionadas pueden obtenerse en el trabajo antes citado.

Se consideraron tres provincias del Noroeste Argentino: Jujuy (complejo de Vilama, lagunas Pozuelos, Los Enamorados y Runtuyoc), Salta (laguna Pastos Grandes) y Catamarca (lagunas La Alumbrera, Purulla, Grande, Carachi Pampa, Diamante, Baya, del Salitre, Blanca y cola del embalse Cortaderas). La división de la laguna Palar en dos cuerpos de agua debido a una reducción volumétrica nos llevó a la toma de una muestra en cada sitio, las cuales fueron denominadas Palar Chica y Palar Grande (Tabla 1, Fig. 1).

$\mathrm{El} \mathrm{pH}$ y la conductividad eléctrica $(\mu \mathrm{S} /$ $\mathrm{cm})$ se midieron in situ con un peachímetro

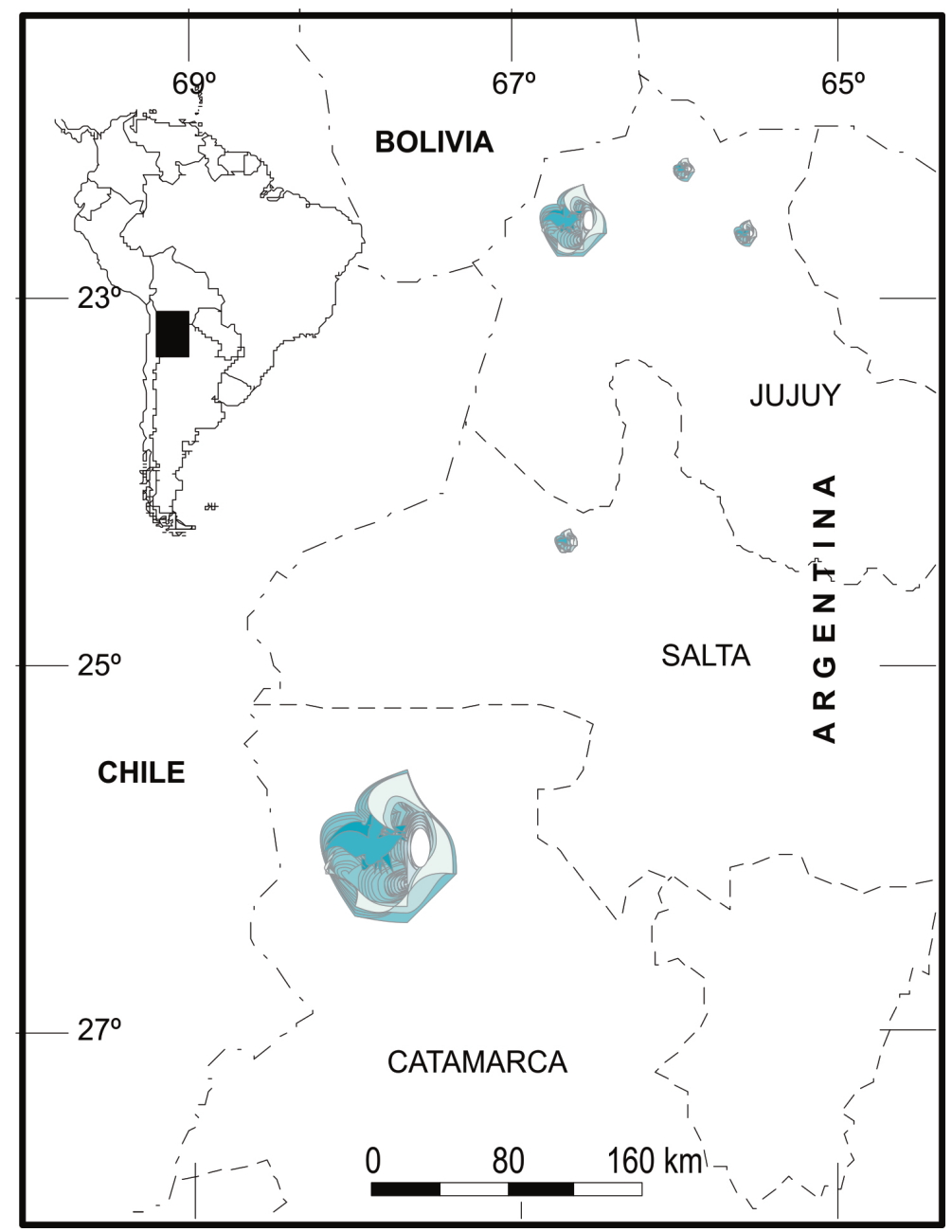

Fig. 1. Ubicación de las áreas de estudio en las provincias de Jujuy, Salta y Catamarca. 
Tabla 1. Ubicación y características ambientales de las lagunas estudiadas.

\begin{tabular}{|c|c|c|c|c|c|c|c|c|}
\hline & Sitios & Latitud & Longitud & Altura (msnm) & Superficie (ha) & $\mathrm{pH}$ & Conductividad $(\mu \mathrm{S} / \mathrm{cm})$ & Salinidad \\
\hline $\mathrm{J} \mathrm{s} 1$ & Laguna Pozuelos & $22^{\circ} 20^{\prime} 29^{\prime \prime} \mathrm{S}$ & $65^{\circ} 57^{\prime} 20^{\prime \prime} \mathrm{W}$ & 3504 & $16470^{* *}$ & 8 & 2000 & salina \\
\hline $\mathrm{J}$ s2 & Laguna Isla Grande & $22^{\circ} 36^{\prime} 21^{\prime \prime} \mathrm{S}$ & $66^{\circ} 48^{\prime} 23^{\prime \prime} \mathrm{W}$ & 4400 & $450^{*}$ & 9 & $8050^{*}$ & salina \\
\hline J s3 & Laguna Catal & $22^{\circ} 42^{\prime} 01^{\prime \prime} \mathrm{S}$ & $66^{\circ} 42^{\prime} 08^{\prime \prime} \mathrm{W}$ & 4320 & $1080^{*}$ & 9 & $40300^{*}$ & salina-hipersalina \\
\hline $\mathrm{J} \mathrm{s} 4$ & Laguna Arenal & $22^{\circ} 40^{\prime} 14^{\prime \prime} \mathrm{S}$ & $66^{\circ} 41^{\prime} 58^{\prime \prime} \mathrm{W}$ & 4631 & $1620^{*}$ & 10 & $22500^{*}$ & salina-hipersalina \\
\hline $\mathrm{J} \mathrm{s5}$ & Laguna Cerro Negro & $22^{\circ} 30^{\prime} 44^{\prime \prime} \mathrm{S}$ & $66^{\circ} 41^{\prime} 31^{\prime \prime} \mathrm{W}$ & 4400 & $900^{*}$ & 8 & $1270^{*}$ & salina \\
\hline $\mathrm{J} \mathrm{s6}$ & Laguna Pululos & $22^{\circ} 30^{\prime} 44^{\prime \prime} \mathrm{S}$ & $66^{\circ} 47^{\prime} 53^{\prime \prime} \mathrm{W}$ & 4413 & $990^{*}$ & 8 & 1380 & salina \\
\hline J s7 & Laguna Caití & $22^{\circ} 32^{\prime} 43^{\prime \prime} \mathrm{S}$ & $66^{\circ} 45^{\prime} 50^{\prime \prime} \mathrm{W}$ & 4573 & $180^{*}$ & 9 & $1150^{*}$ & salina \\
\hline $\mathrm{J} \mathrm{s} 8$ & Laguna Guinda & $22^{\circ} 47^{\prime} 55^{\prime \prime} \mathrm{S}$ & $66^{\circ} 50^{\prime} 22^{\prime \prime} \mathrm{W}$ & \pm 4410 & $<70$ & 11 & 123760 & hipersalina \\
\hline $\mathrm{J} \mathrm{s} 9$ & Laguna Honda & $22^{\circ} 49^{\prime} 11^{\prime \prime} \mathrm{S}$ & $66^{\circ} 51^{\prime} 01^{\prime \prime} \mathrm{W}$ & \pm 4410 & $<70$ & 11 & 116880 & hipersalina \\
\hline J $\mathrm{s} 10$ & Laguna Blanca & $22^{\circ} 50^{\prime} 10^{\prime \prime} \mathrm{S}$ & $66^{\circ} 55^{\prime} 07^{\prime \prime} \mathrm{W}$ & \pm 4410 & $<70$ & 11 & 130560 & hipersalina \\
\hline $\mathrm{J} \quad \mathrm{s} 11$ & Laguna Vilama & $22^{\circ} 36^{\prime} 21^{\prime \prime} \mathrm{S}$ & $66^{\circ} 55^{\prime} 23^{\prime \prime} \mathrm{W}$ & 4400 & $4590^{*}$ & 8 & $268800^{*}$ & hipersalina \\
\hline J $\mathrm{s} 12$ & Laguna Colpayoc & $22^{\circ} 39^{\prime} 50^{\prime \prime} \mathrm{S}$ & $66^{\circ} 51^{\prime} 36^{\prime \prime} \mathrm{W}$ & 4389 & $180^{*}$ & 10 & $2960^{*}$ & salina \\
\hline $\mathrm{J} \mathrm{s} 13$ & Laguna Palar Chica & $22^{\circ} 40^{\prime} 24^{\prime \prime} \mathrm{S}$ & $66^{\circ} 48^{\prime} 41^{\prime \prime} \mathrm{W}$ & 4309 & $2250^{*}$ & 8 & $108300^{*}$ & hipersalina \\
\hline J $\quad$ s14 & Laguna Palar Grande & $22^{\circ} 40^{\prime} 24^{\prime \prime} \mathrm{S}$ & $66^{\circ} 48^{\prime} 41^{\prime \prime} \mathrm{W}$ & 4309 & $2250^{*}$ & 8 & $108300^{*}$ & hipersalina \\
\hline J $\mathrm{s} 15$ & Laguna Runtuyoc & $22^{\circ} 39^{\prime} 31^{\prime \prime} \mathrm{S}$ & $65^{\circ} 41^{\prime} 33^{\prime \prime} \mathrm{W}$ & 3482 & $<70^{* *}$ & 8 & 1516 & salina \\
\hline J s16 & Laguna Los Enamorados & $22^{\circ} 43^{\prime} 30^{\prime \prime} \mathrm{S}$ & $65^{\circ} 41^{\prime} 30^{\prime \prime} \mathrm{W}$ & 3482 & $<70^{* *}$ & 7 & 8700 & salina \\
\hline S s17 & Laguna Pastos Grandes & $24^{\circ} 33^{\prime} 18^{\prime \prime} \mathrm{S}$ & $66^{\circ} 40^{\prime} 05^{\prime \prime} \mathrm{W}$ & 3900 & $<70^{* *}$ & 9 & 405840 & hipersalina \\
\hline C s18 & Laguna La Alumbrera & $26^{\circ} 06^{\prime} 46^{\prime \prime} \mathrm{S}$ & $67^{\circ} 25^{\prime} 13^{\prime \prime} \mathrm{W}$ & 3250 & $217^{\star *}$ & 9,8 & 1450 & salina \\
\hline C $\$ 19$ & Laguna Purulla & $26^{\circ} 40^{\prime} 26^{\prime \prime S}$ & $67^{\circ} 41^{\prime} 15^{\prime \prime} \mathrm{W}$ & 3664 & $144^{\star *}$ & 8,4 & 61160 & salina-hipersalina \\
\hline C s20 & Laguna Grande & $26^{\circ} 13^{\prime} 38^{\prime \prime} \mathrm{S}$ & $67^{\circ} 03^{\prime} 40^{\prime \prime} \mathrm{W}$ & 4101 & $433^{\star \star}$ & 8,8 & 6950 & salina \\
\hline C $s 21$ & Laguna Carachi Pampa & $26^{\circ} 26^{\prime} 54^{\prime \prime} \mathrm{S}$ & $67^{\circ} 30^{\prime} 23^{\prime \prime} \mathrm{W}$ & 2915 & $361^{\star \star}$ & 7,9 & 498240 & hipersalina \\
\hline C s22 & Laguna Diamante & $26^{\circ} 02^{\prime} 07^{\prime \prime} \mathrm{S}$ & $67^{\circ} 01^{\prime} 25^{\prime \prime} \mathrm{W}$ & 4388 & $1372^{* *}$ & 8,9 & 481600 & hipersalina \\
\hline C s23 & Laguna Baya & $26^{\circ} 14^{\prime} 16^{\prime \prime} \mathrm{S}$ & $66^{\circ} 59^{\prime} 10^{\prime \prime} \mathrm{W}$ & 4200 & $72^{* *}$ & 9,5 & 176640 & hipersalina \\
\hline C s24 & Laguna del Salitre & $26^{\circ} 15^{\prime} 05^{\prime \prime} \mathrm{S}$ & $66^{\circ} 54^{\prime} 04^{\prime \prime} \mathrm{W}$ & 4082 & sd & $\mathrm{sd}$ & 514 & salina \\
\hline C s25 & Laguna Blanca & $26^{\circ} 38^{\prime} 02^{\prime \prime} \mathrm{S}$ & $66^{\circ} 56^{\prime} 13^{\prime \prime} \mathrm{W}$ & 3147 & $1372^{\star \star}$ & 8,5 & 612 & salina \\
\hline C s26 & Embalse Cortaderas (cola) & $27^{\circ} 01^{\prime} 51^{\prime \prime} \mathrm{S}$ & $68^{\circ} 08^{\prime} 64^{\prime \prime} \mathrm{W}$ & 3900 & sd & 8,3 & 1345 & salina \\
\hline
\end{tabular}

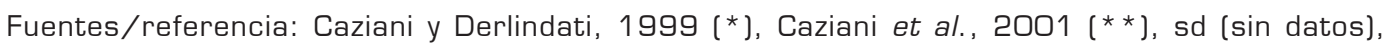
C (Catamarca), J (Jujuy), S [Salta).

digital portátil y un conductímetro de lectura directa. En relación con la profundidad de las lagunas, Caziani y Derlindati (2000) definieron tres tipos en base a sus pendientes que fueron estimadas en el centro de sus cubetas: somera (menor de 1\%), intermedia (1\%) y profunda (mayor de $2 \%$ ).

Las muestras cualitativas se obtuvieron mediante el filtrado de 25 litros de agua a través de una red de $20 \mu \mathrm{m}$ de poro y fueron fijadas in situ con formaldehído al 4\%. Ante la poca profundidad de la mayoría de estos ambientes leníticos hubo que introducirse a varios metros de las orillas para la extracción del material ficológico. Se empleó un microscopio binocular con dispositivo para dibujo para la observación de los ejemplares a diferentes aumentos. Las medidas de las especies son propias, colocándose entre paréntesis las menos frecuentes.

Para la elaboración de este trabajo se consultó a Bourrelly $(1972,1985)$, Comas González (1996), Huber-Pestalozzi (1961, 1983), Krieger (1937), Tell y Conforti (1986), Tell et al. (1994), Tracanna (1981, 1985), Uherkovich (1966), Van den Hoek et al. (1995). En referencia a las distribuciones geográficas de las especies se recurrió a los catálo- gos de Tell (1985) y Del Giorgio (1988) y a publicaciones del Noroeste Argentino, esto último con la finalidad de conocer las especies citadas por primera vez para esta región de acuerdo a los trabajos consultados. Los nombres de los lugares donde las entidades taxonómicas fueron localizadas se colocaron alfabéticamente. Otras citas bibliográficas son indicadas en Taxonomía y Discusión.

Con referencia al material estudiado, las características de los ambientes y las distribuciones de las especies en los sitios de muestreo son detalladas en las tablas 1 y 2 .

\section{RESULTADOS}

En los cuerpos leníticos seleccionados, de aguas alcalinas y salinidades variables (Tabla 1 ), se reconocieron 28 taxones pertenecientes a clorofitas (24) y euglenofitas (4). El número más alto de especies fue ocho en Los Enamorados, una en Grande, Isla Grande y Blanca-Cat y sin registro en el $26 \%$ de los cuerpos de agua incluidos en este trabajo (Tabla 2, Fig. 2).

Las clorofitas, ausentes en Guinda, Honda, Blanca, Pastos Grandes y Purulla variaron de una en Isla Grande, Grande, Catal y Blan- 


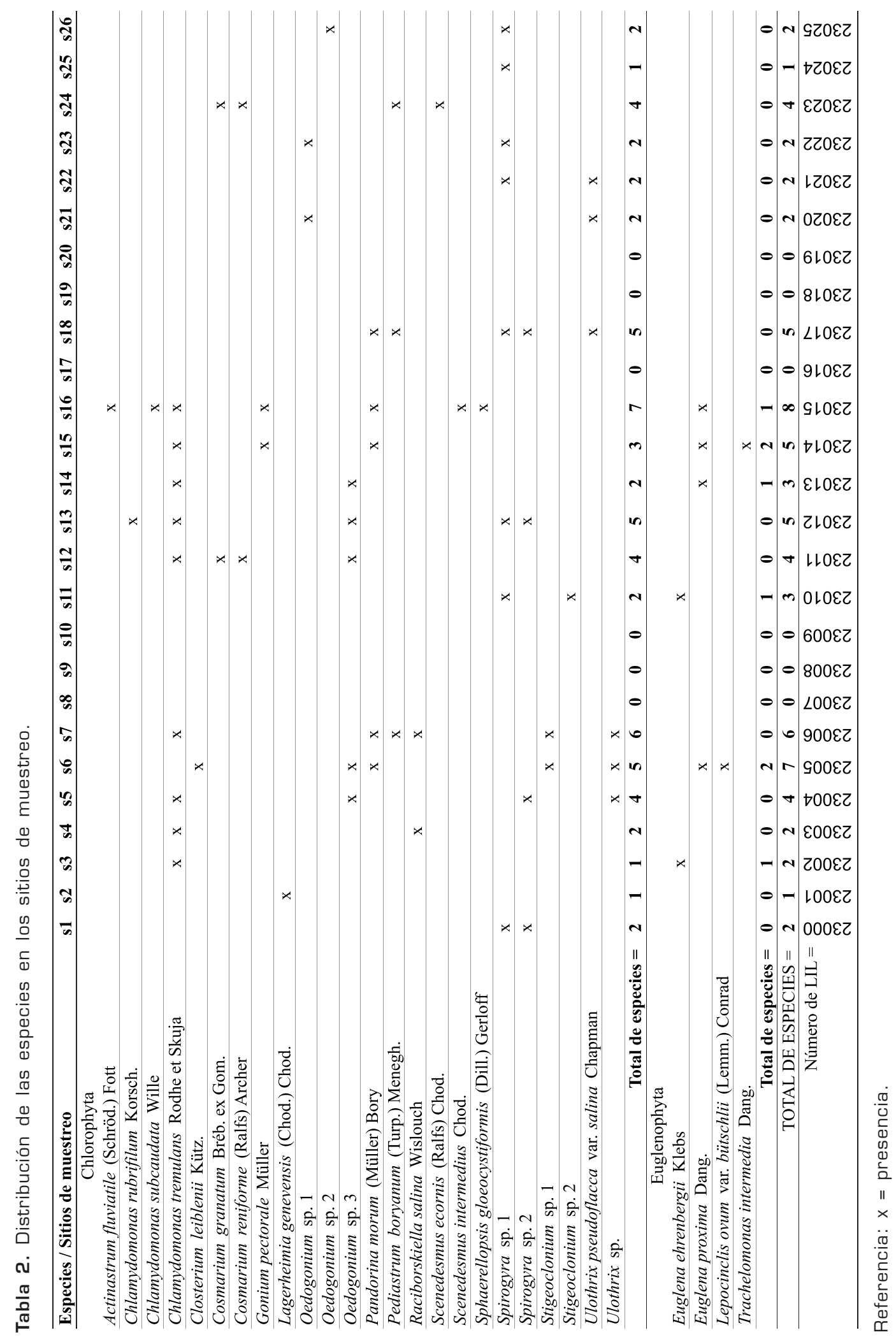




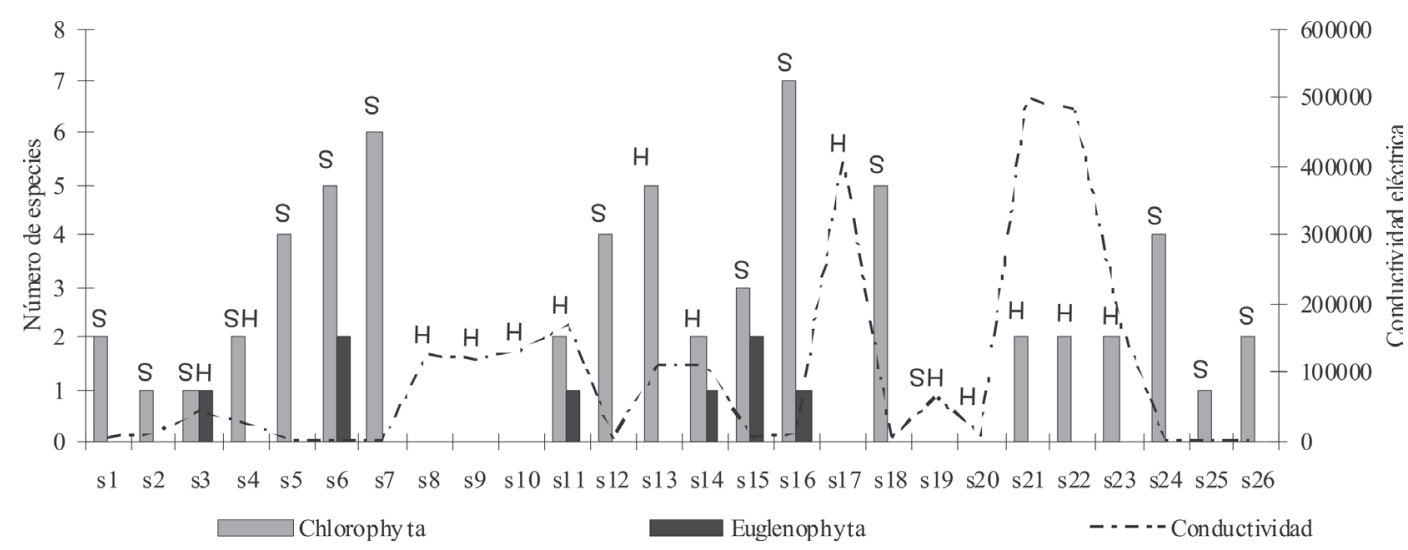

Fig. 2. Distribución de la riqueza en relación a la conductividad eléctrica (referencia: S, salina; $\mathrm{SH}$, salina-hipersalina; $\mathrm{H}$, hipersalina).

ca-Cat hasta siete especies en Los Enamorados y La Alumbrera. Dentro de este grupo el taxón que más aportó a nivel infragenérico fue Chlamydomonas sp.

Las euglenofitas, observadas en seis de los veintiséis puntos seleccionados, sólo fueron detectadas en la provincia de Jujuy y en niveles muy bajos de hasta dos especies en las lagunas Pululos y Runtuyoc, una en Catal, Vilama, Palar Grande y Los Enamorados y sin datos en los ambientes restantes.

Las entidades taxonómicas encontradas en más de un sitio fueron Chlamydomonas tremulans, Oedogonium sp., Pediastrum boryanum, Pandorina morum, Spirogyra sp. 1, Spirogyra sp. 2, Ulothrix pseudoflacca var. salina, Ulothrix sp. y Euglena proxima, las cuales alcanzaron frecuencias superiores al $11 \%$, mientras que las otras fluctuaron entre porcentajes del 3,8-7,7. Los taxones Actinastrum fluviatile, Chlamydomonas rubrifilum, Chlamydomonas subcaudata, Closterium leiblenii, Lagerheimia genevensis, Oedogonium sp. 2, Scenedesmus intermedius, Sphaerellopsis gloeocystiformis, Stigeoclonium sp., Lepocinclis ovum var. bütschlii y Trachelomonas intermedia fueron registrados en un único punto de muestreo.

En relación a la tolerancia salina de acuerdo a los datos de campo, diecisiete de las veintiocho especies encontradas en los cuerpos leníticos estudiados, es decir el $60,7 \%$, estuvieron en ambientes salinos.
Chlamydomonas rubrifilum, Oedogonium sp. 1 y Stigeoclonium sp. 2 fueron las únicas halladas sólo en aguas hipersalinas, no así Chlamydomonas tremulans, Oedogonium sp. 3, Spirogyra sp. 1, Spirogyra sp. 2, Ulothrix pseudoflacca var. salina, Euglena ehrenbergii y Euglena proxima que también fueron observadas a salinidades menores. En el caso de Raciborskiella salina fue detectada en aguas salinas como salinas-hipersalinas con conductividades de hasta $22500 \mu \mathrm{S} / \mathrm{cm}$.

\section{TAXONOMÍA}

División Chlorophyta

Clase Chlorophyceae

Orden Volvocales

Familia Chlamydomonadaceae

Chlamydomonas Ehrenberg, 1833

Chlamydomonas rubrifilum

Korschikoff, in Pascher, Süssw.-Fl.

Deutschl. 4, p. 188, 1927.

(Fig. 3A)

Célula ampliamente elíptica a cilíndrica-elíptica, con papila cuneiforme-roma pequeña y extremo posterior redondeado, de 10-24 $\mu \mathrm{m}$ de longitud y 6-14 $\mu \mathrm{m}$ de ancho. Cromatóforo cupuliforme, 8-10 pirenoides dispersos y estigma.

Ecología.- En turberas y otros ambientes (Huber-Pestalozzi, 1961). Aeroterrestre y de agua dulce (Klochkova et al., 2008). 
Distribución geográfica.- En Argentina: sin datos (Tell, 1985; Del Giorgio, 1988). En el Noroeste Argentino: Jujuy (Mirande y Tracanna, 2007).

Chlamydomonas subcaudata Wille, Nyt. Mag. for Naturvid. 41, p. 118, 1903.

(Fig. 3B)

Célula elíptica-alargada, con papila subhemisférica y extremo posterior levemente angostado e incoloro, de (9) 13-15 $\mu \mathrm{m}$ de longitud y 6-7 $\mu \mathrm{m}$ de ancho. Cromatóforo cupuliforme, estriado, con un pirenoide central y estigma.

Ecología.- Aguas estancadas, pantanos (Huber-Pestalozzi, 1961).

Distribución geográfica.- En Argentina: sin datos (Tell, 1985; Del Giorgio, 1988). En el Noroeste Argentino: Jujuy (Mirande y Tracanna, 2007).

Chlamydomonas tremulans

Rhode et Skuja, Symbolae Bot. Ups. 9 (3), p. 84, 1948.

(Fig. 3C)

Célula elíptica, con papila aguzada y extremo posterior redondeado, de 6,5-11,5 $\mu \mathrm{m}$ de longitud y 4-8,5 $\mu \mathrm{m}$ de ancho. Cromatóforo cupuliforme, con un pirenoide central y estigma.

Ecología. - En aguas marinas, en el plancton (Huber-Pestalozzi, 1961).

Distribución geográfica.- En Argentina: sin datos (Tell, 1985; Del Giorgio, 1988). En el Noroeste Argentino: Jujuy (Mirande y Tracanna, 2007).

Sphaerellopsis Korschikoff, 1925

Sphaerellopsis gloeocystiformis (Dill.) Gerloff, Arch. Protistenk. 94, p. 486, 1940. (Fig. 3D)

Especie caracterizada por la presencia de mucílago entre la pared celular y plasmalema. Célula (protoplasto y cubierta mucilaginosa) ampliamente ovalada a brevemente elíptica. Protoplasto ovalado a piriforme, con papila evidente o no, aguzada o roma, de 13-15 $\mu \mathrm{m}$ de longitud y $11-13 \mu \mathrm{m}$ de ancho. Cromatóforo cupuliforme, no estriado, con un pirenoide central y estigma.

Ecología.- En cultivos, suelo, entre otros ambientes (Huber-Pestalozzi, 1961).

Distribución geográfica.- En Argentina: sin datos (Tell, 1985; Del Giorgio, 1988). En el Noroeste Argentino: Jujuy (Mirande y Tracanna, 2007).

Familia Polyblepharidaceae

Raciborskiella Wislouch, 1924

Raciborskiella salina Wislouch, Act. Soc. Bot. Pol. 2, p. 99-129, 1924.

(Fig. 3E)

Colonia compacta. Células esféricas, de 5,5-7,5 $\mu \mathrm{m}$ de longitud y 3-4 $\mu \mathrm{m}$ de ancho, sin apéndice caudal. Cromatóforo cupuliforme, con un pirenoide basal y estigma.

Observaciones. - Ejemplares más pequeños que el tipo. Huber-Pestalozzi (1961) cita para la especie una longitud de 11-15 $\mu \mathrm{m}$ y ancho de 4-8 $\mu \mathrm{m}$.

Ecología.- En aguas salobres (HuberPestalozzi, 1961).

Distribución geográfica.- En Argentina: sin datos (Tell, 1985; Del Giorgio, 1988). En el Noroeste Argentino: Jujuy (Mirande y Tracanna, 2007).

Familia Volvocaceae Gonium Müller, 1773

Gonium pectorale Müller, Vermium Hist., Lipsiae, p. 60, 1773.

(Fig. 3F)

Colonia plana, formada por 16 células (4 céntricas y 12 periféricas), de 37-45 $\mu \mathrm{m}$ de longitud. Células elípticas a ovales, de (7) 10-12 $\mu \mathrm{m}$ de longitud y (7) 9-10 $\mu \mathrm{m}$ de ancho. Cromatóforo cupuliforme, con un pirenoide basal.

Ecología.- En aguas estancadas y fluyentes (Huber-Pestalozzi, 1961), en el plancton (Margalef, 1983).

Distribución geográfica.- En Argentina: Buenos Aires, Córdoba, Tierra del Fuego (Tell, 1985); sin datos (Del Giorgio, 1988). 

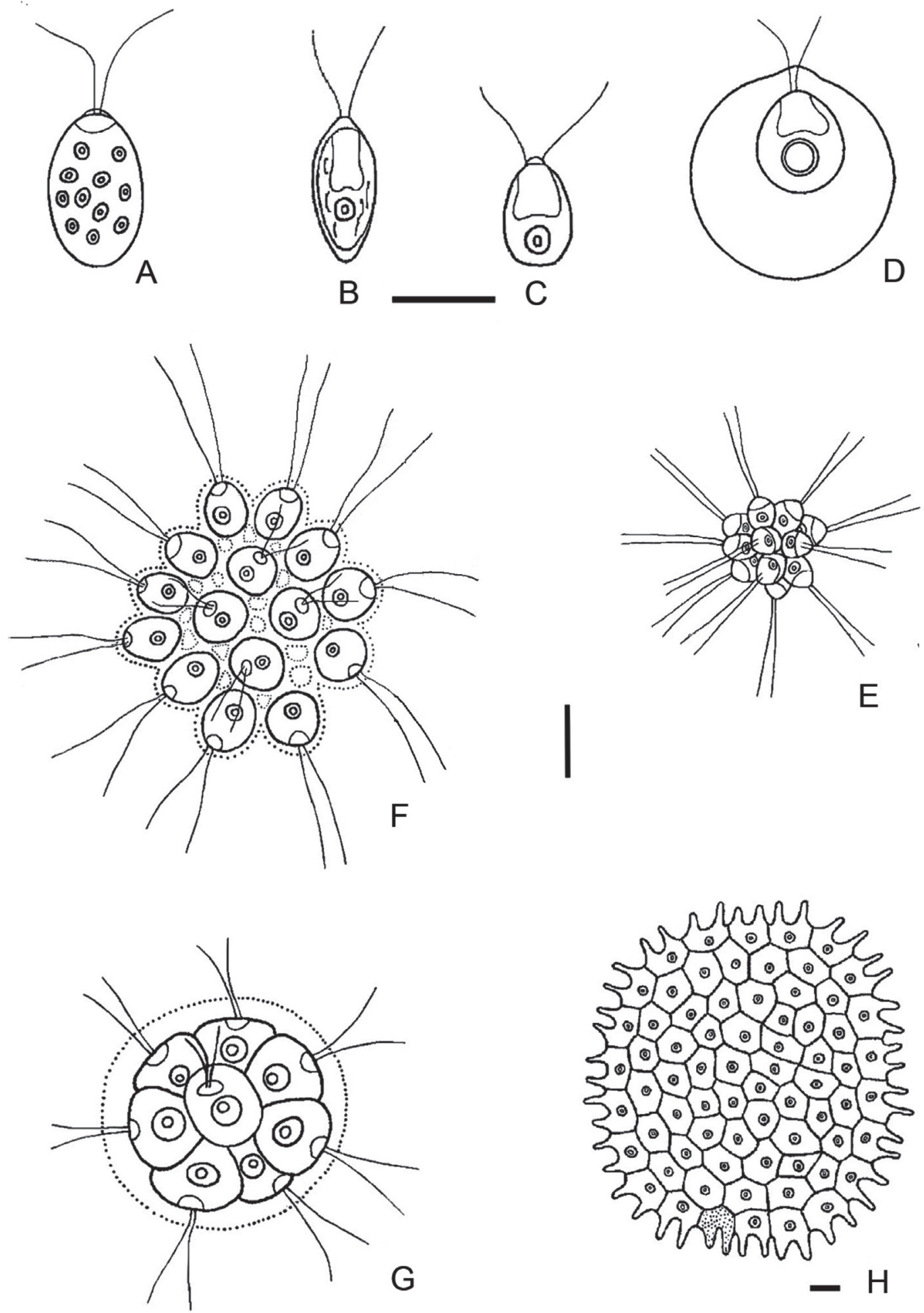

Fig. 3. A) Chlamydomonas rubrifilum. B) Chlamydomonas subcaudata. C) Chlamydomonas tremulans. D) Sphaerellopsis gloeocystiformis. E) Raciborskiella salina. F) Gonium pectorale. G) Pandorina morum. H] Pediastrum boryanum. Las escalas equivalen a $10 \mu \mathrm{m}$. 
En el Noroeste Argentino: Jujuy (Mirande y Tracanna, 2007); Salta (Tracanna, 1985); Tucumán (Tracanna, 1981).

Pandorina Bory, 1824

Pandorina morum (Müll.) Bory,

Encycl. Method. Zooph., p. 521, 1825.

(Fig. 3G)

Colonias elipsoidales, a veces esféricas, con 8-16 células dentro de una vaina mucilaginosa, de 28-50 $\mu \mathrm{m}$ de longitud y 24-40 $\mu \mathrm{m}$ de ancho. Células piriformes-angulares, biflageladas, de 8-18 $\mu \mathrm{m}$ de longitud y 7-17 (25) $\mu \mathrm{m}$ de ancho.

Ecología. - Cosmopolita (Kammerer, 1938; Tracanna, 1981). En ambientes acuáticos eutróficos, oligotróficos, en el mar (Huber-Pestalozzi, 1961), en el plancton (Margalef, 1983).

Distribución geográfica.- En Argentina: Buenos Aires, Córdoba, Chubut, Entre Ríos, Neuquén, Patagonia, Río Negro, Santa Cruz, Tierra del Fuego (Tell, 1985); sin datos (Del Giorgio, 1988). En el Noroeste Argentino: Catamarca y Jujuy (Tracanna, 1985); Salta (Tracanna, 1985; Salusso, 1998); Tucumán (Tracanna, 1981, 1985; Seeligmann y Tracanna, 1994; Mirande y Tracanna, 1995, 2004; Tracanna et al., 1996, 1999; Seeligmann, 1998; Seeligmann et al., 2001).

Orden Chlorococcales

Familia Hydrodictyaceae

Pediastrum Meyen, 1829

Pediastrum boryanum var. boryanum (Turp.) Meneghini, Synops. Linnaea 14, p. 210, 1840.

(Fig. 3H)

Colonias de 4-64 (raro 128) células poligonales dispuestas en series concéntricas, que no dejan espacios intercelulares o son diminutos. Células internas de (5) 10-15 (20) $\mu \mathrm{m}$ de longitud y (5) $10-22$ (27) $\mu \mathrm{m}$ de ancho. Células marginales de (5) 7-15 (17) $\mu \mathrm{m}$ de longitud y 10-20 (27) $\mu \mathrm{m}$ de ancho, con dos procesos simples, truncados, dispuestos en el mismo plano, cortos o hasta
$8 \mu \mathrm{m}$ de largo. Escultura de la pared puntiforme.

Ecología.- Cosmopolita. Muy adaptable a diferentes ambientes, frecuente en aguas moderadamente ácidas, al igual que en neutras a levemente alcalinas, en la mayoría de los casos eutróficas (Parra Barrientos, 1979); en el plancton (Huber-Pestalozzi, 1983).

Distribución geográfica. - En Argentina: Buenos Aires, Córdoba, Corrientes, Chubut, La Rioja, Malvinas, Patagonia, Río Negro, Tierra del Fuego (Tell, 1985); sin datos (Del Giorgio, 1988). En el Noroeste Argentino: Catamarca (Tracanna, 1985); Jujuy (Mirande y Tracanna, 2007); Tucumán (Tracanna, 1981, 1985).

Familia Micractiniaceae Lagerheimia Chodat, 1895

Lagerheimia genevensis (Chod.) Chodat, Nuova Notarisia 6, p. 90, 1895.

(Fig. 4A)

Células cilíndricas a ovales o elípticas, solitarias, libres, de 7-10 $\mu \mathrm{m}$ de longitud y 3,5-6,5 (8) $\mu \mathrm{m}$ de ancho, y de extremos redondeados. Presencia de dos sedas polares subapicales con una protuberancia basal, divergentes, en los polos, de 14-20 $\mu \mathrm{m}$ de longitud. Cromatóforo parietal con un pirenoide.

Ecología.- Cosmopolita. Planctónica, en aguas dulces, estancadas y fluyentes, límpidas a eutróficas, marinas (Huber-Pestalozzi, 1983).

Distribución geográfica.- En Argentina: Buenos Aires, Córdoba (Tell, 1985, como Chodatella quadriseta Lemm.); sin datos (Del Giorgio, 1988). En el Noroeste Argentino: Jujuy (Mirande y Tracanna, 2007); Tucumán (Tracanna, 1981, como C. quadriseta Lemm.).

Familia Scenedesmaceae Actinastrum Lagerheim, 1888 Actinastrum fluviatile (Schröd.) Fott, Preslia 49, p. 6, 1977.

(Fig. 4B)

Colonias de 4-8 células dispuestas en más de un plano. Células fusiformes, de extremos 
aguzados a levemente redondeados, de 3,5$14 \mu \mathrm{m}$ de longitud y $2,7-3 \mu \mathrm{m}$ de ancho. Cromatóforo parietal con un pirenoide central.

Ecología.- En aguas dulces y marinas, a veces en las planicies de inundación de grandes ríos, planctónica (Huber-Pestalozzi, 1983).

Distribución geográfica.- En Argentina: sin datos (Tell, 1985; Del Giorgio, 1988). En el Noroeste Argentino: Jujuy (Mirande y Tracanna, 2007).

Scenedesmus Meyen, 1829

Scenedesmus ecornis (Ralfs) Chodat, Scenedesmus. Étude de génétique etc., p. 170, fig. 60, 1926.

(Fig. 4C)

Colonias lineares de 2, 4, 8 a veces 16 y hasta 32 células elipsoidales dispuestas en una serie. Células de L: 8-10 $\mu \mathrm{m}$ y 1: 3-4 $\mu \mathrm{m}$.

Ecología.- Cosmopolita (Comas González, 1996). Especie beta-mesosaprobionte (Uherkovich, 1966).

Distribución geográfica.- En Argentina: en Tell (1985), Buenos Aires, Neuquén, Río Negro. En el NOA: Catamarca (Tracanna, 1985, como Sc. obtusus f. ecornis (Ehr.) Compère), Salta (Moraña, 1998; Salusso, 1998), Tucumán (Seeligmann y Tracanna, 1994; Martínez De Marco, 1995; Tracanna y Martínez De Marco, 1997).

Scenedesmus intermedius Chodat, Z. Hydrol. 3, p. 231, 1926.

(Fig. 4D)

Colonias planas de 2-4-8 células elípticas u ovales dispuestas alternadamente. Células de 12-12,5 $\mu \mathrm{m}$ de longitud y 4,5-5 $\mu \mathrm{m}$ de ancho. Espinas de 1: 7-8 $\mu \mathrm{m}$.

Ecología.- Cosmopolita. Frecuente en acuíferos eutróficos de las provincias occidentales cubanas (Comas González, 1996). En ambientes eutróficos, aguas dulces y marinas, planctónica (Huber-Pestalozzi, 1983).

Distribución geográfica. - En Argentina: Buenos Aires, Santa Fe (Tell, 1985); sin da- tos (Del Giorgio, 1988). En el Noroeste Argentino: Jujuy (Mirande y Tracanna, 2007); Salta (Salusso, 1998); Tucumán (Tracanna, 1981; Mirande y Tracanna, 1995, 2004; Tracanna et al., 1996).

\section{Orden Chaetophorales \\ Stigeoclonium Kützing, 1843 \\ Stigeoclonium sp. 1}

Filamentos erguidos con ramificación dicotómica-alterna. Células de las ramas primarias y secundarias de 1: (6) 9-15 (20) $\mu \mathrm{m}$ de longitud y 5-7 (8) $\mu \mathrm{m}$ de ancho.

Observaciones. - Material poco frecuente e incompleto.

\section{Stigeoclonium sp. 2}

Filamentos erguidos poco ramificados. Células del eje postrado, de (5) 8-13 (20) $\mu \mathrm{m}$ de longitud y (7) $12-15$ (28) $\mu \mathrm{m}$ de ancho. Células de las ramas primarias y secundarias, de (9) 12-32 (60) $\mu \mathrm{m}$ de longitud y (3) 7-11 (12) $\mu \mathrm{m}$ de ancho, algunas terminadas en un pelo hialino de (62) 115-130 $\mu \mathrm{m}$ de largo.

Observaciones. - Material poco frecuente e incompleto.

\section{Orden Oedogoniales \\ Oedogonium Link, 1820 \\ Oedogonium sp. 1}

Células en forma de tonel, de (30) 40-47 (52) $\mu \mathrm{m}$ de longitud y (15) $17-18$ (20) $\mu \mathrm{m}$ de ancho. Célula apical levemente atenuadaredondeada. Célula basal pequeña.

Observaciones. - Material estéril, incompleto, poco frecuente.

\section{Oedogonium sp. 2}

Células en forma de tonel, de (60) 70-77 (81) $\mu \mathrm{m}$ de longitud y 25-27 (30) $\mu \mathrm{m}$ de ancho. Oogonio de d: $55 \mu \mathrm{m}$. Célula apical levemente atenuada-redondeada. Célula basal pequeña.

Observaciones. - Material estéril, incompleto, poco frecuente. 


\section{Oedogonium sp. 3}

Células en forma de tonel, de 15-30 $\mu \mathrm{m}$ de longitud y 6-8 (9) $\mu \mathrm{m}$ de ancho. Célula apical levemente atenuada-redondeada. Célula basal pequeña.

Observaciones. - Material estéril, incompleto, poco frecuente.

\section{Clase Ulvophyceae \\ Orden Codiolales \\ Ulothrix Kützing, 1833 \\ Ulothrix sp.}

Filamentos no ramificados, libremente flotantes, uniseriados. Células en forma de tonel, subcuadradas o levemente más largas que anchas, de $(2,5) 3-4 \mu \mathrm{m}$ de longitud y 3-3,5 $\mu \mathrm{m}$ de ancho, con cloroplasto parietal en forma de faja que rodea más de la mitad de la célula, un pirenoide y pared delgada.

Observaciones. - Material poco frecuente.

Ulothrix pseudoflacca var. salina

Chapman, Blumea 24: 191-299, 1946.

(Fig. 4E)

Filamentos de $6200 \mu \mathrm{m}$ de longitud y $15 \mu \mathrm{m}$ de ancho. Células de 7-8 (9) $\mu \mathrm{m}$ de longitud y 10-12 $\mu \mathrm{m}$ de ancho. Cloroplasto parietal con un pirenoide central.

Ecología.- En aguas salinas y marinas, en un rango de salinidad entre 7,9-9,1\% (Ramanathan, 1964).

Distribución geográfica.- En Argentina: sin datos (Tell, 1985; Del Giorgio, 1988). En el Noroeste Argentino: sin datos.

\section{Clase Zygnematophyceae Orden Zygnematales Spirogyra Link, 1820 Spirogyra sp. 1}

Células cilíndricas de 64-170 (200) $\mu \mathrm{m}$ de longitud y (50) 52-60 $\mu \mathrm{m}$ de ancho. Dos a tres cloroplastos cintados. Tabiques transversales planos.

Observaciones. - Material estéril.
Spirogyra sp. 2

Células cilíndricas de (75) 120-145 (255) $\mu \mathrm{m}$ de longitud y (15) 20-25 (30) $\mu \mathrm{m}$ de ancho. Un cloroplasto cintado. Tabiques transversales planos.

Observaciones. - Material estéril.

$$
\begin{gathered}
\text { Orden Desmidiales } \\
\text { Familia Closteriaceae } \\
\text { Closterium Nitzsch, } 1817 \\
\text { Closterium leibleinii Kützing, in Ralfs, } \\
\text { Brit. Desm., p. 167, 1848. }
\end{gathered}
$$

(Fig. 4F)

Células siete veces más largas que anchas, de 120-165 $\mu \mathrm{m}$ de longitud y 18-24 $\mu \mathrm{m}$ de ancho. Márgenes externo convexo e interno dilatado en el medio, atenuados gradualmente hacia los ápices. Extremos redondeados, de 3-5 $\mu \mathrm{m}$ de ancho. Cloroplastos con 5-6 pirenoides por hemicélula. Vacuolas apicales con 8-12 gránulos. Pared celular lisa, incolora, sin bandas de crecimiento.

Ecología.- Cosmopolita (Lacoste de Díaz, 1979; Tell et al., 1994). Aguas estancadas y fluyentes eutróficas, sulfurosas, etc. (Krieger, 1937).

Distribución geográfica.- En Argentina: Antártida, Buenos Aires, Córdoba, Misiones, Río Negro, Santa Cruz, Tierra del Fuego (Tell, 1985); sin datos (Del Giorgio, 1988). En el Noroeste Argentino: Jujuy (Mirande y Tracanna, 2007); Tucumán (Mirande y Tracanna, 2004).

Cosmarium Corda, 1834

Cosmarium granatum Brébisson, in Ralfs, Brit. Desm., p. 46, 1848.

(Fig. 4G)

Hemicélulas truncado-piramidales, lados subparalelos a convexos en la base, vista apical elíptica, de 19-22 $\mu \mathrm{m}$ de longitud y 11$12,5 \mu \mathrm{m}$ de ancho e istmo evidente de 5-6 $\mu \mathrm{m}$ de ancho. Pared de la célula finamente punteada.

Ecología. - Cosmopolita. Se cita un amplio rango de condiciones ecológicas para poblaciones europeas de esta especie, inclu- 


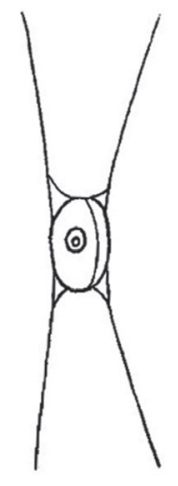

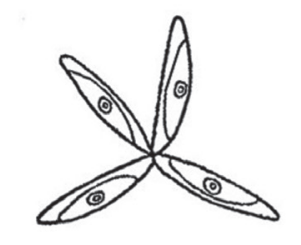

B

A

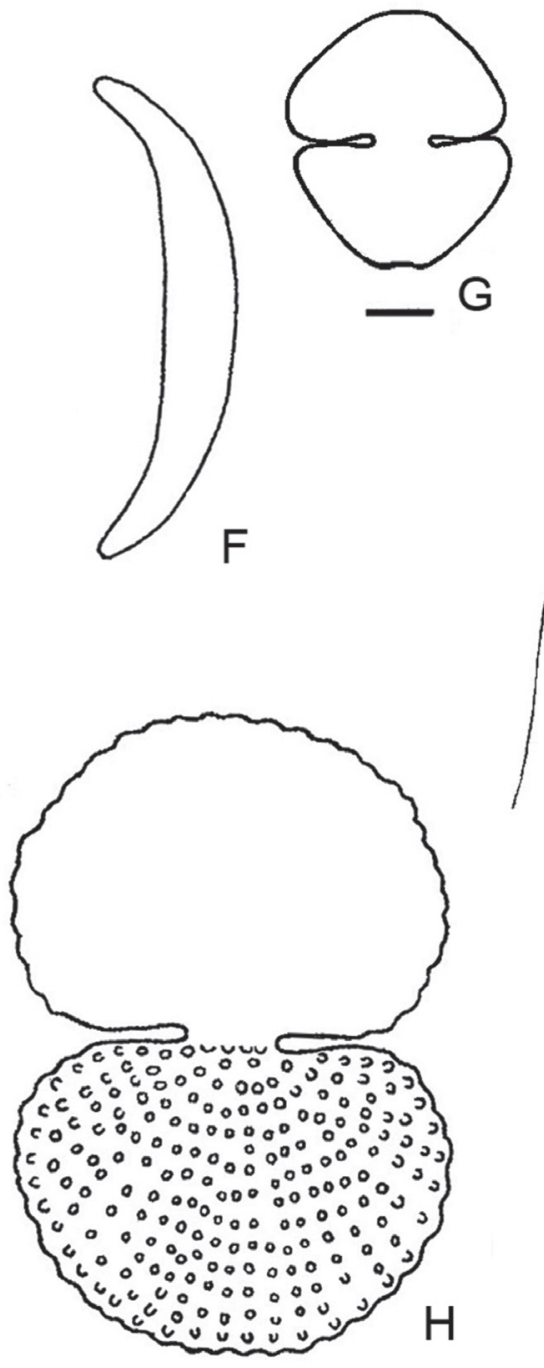

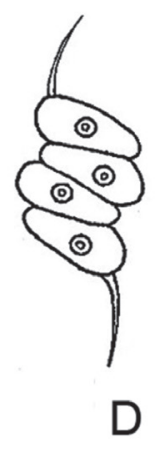

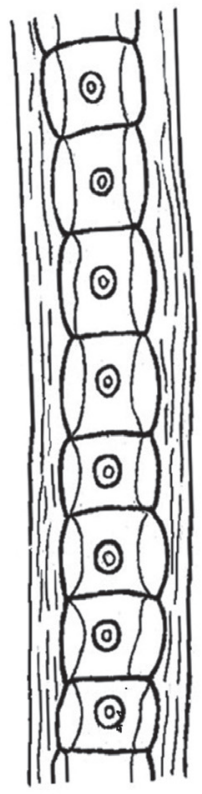

$E$

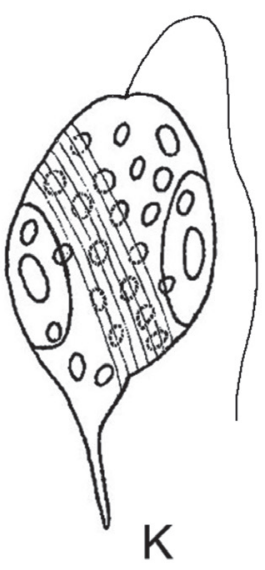

Fig. 4. A) Lagerheimia genevensis. B] Actinastrum fluviatile. C) Scenedesmus ecornis. D] Scenedesmus intermedius. E) Ulothrix pseudoflacca var. salina. F) Closterium leibleinii. G) Cosmarium granatum. H] Cosmarium reniforme. I) Euglena ehrenbergii. J) Euglena proxima. KJ Lepocinclis ovum var. bütschlii. L) Trachelomonas intermedia. Las escalas equivalen a $10 \mu \mathrm{m}$. 
sive en ambientes distróficos y eutróficos, y un rango de $\mathrm{pH}$ que oscila entre 4,6-9,9 (Förster, en Tell et al., 1994).

Distribución geográfica.- En Argentina: Antártida, Buenos Aires, Córdoba, Corrientes, Entre Ríos, La Rioja, Mendoza, Santa Cruz, Tierra del Fuego (Tell, 1985); sin datos (Del Giorgio, 1988). En el Noroeste Argentino: Catamarca (Tracanna, 1985); Jujuy (Mirande y Tracanna, 2007); Tucumán (Tracanna, 1981, 1985).

\section{Cosmarium reniforme (Ralfs) Archer, p. 92, 1874.}

(Fig. 4H)

Hemicélulas reniformes, vista apical elíptica, de 48-65 $\mu \mathrm{m}$ de longitud y 40-44 $\mu \mathrm{m}$ de ancho e istmo estrecho, de 11-13 $\mu \mathrm{m}$ de ancho. Pared de la célula ornamentada con gránulos redondeados y conspicuos, dispuestos oblicuamente o en series verticales.

Ecología.- Cosmopolita (Tell et al., 1994).

Distribución geográfica.- En Argentina: Buenos Aires, Córdoba, delta del Paraná, Jujuy, La Rioja (Tell, 1985); sin datos (Del Giorgio, 1988). En el Noroeste Argentino: Jujuy (Tell, 1985; Mirande y Tracanna, 2007).

\section{División Euglenophyta \\ Clase Euglenophyceae Orden Euglenales \\ Familia Euglenaceae \\ Euglena Ehrenberg, 1830 \\ Euglena ehrenbergii Klebs, Unters. Bot. Inst. Tübing., v. 1-2, p. 304, 1883.}

(Fig. 4I)

Células aplanadas dorsiventralmente o cilíndricas, lados casi paralelos, extremos redondeados siendo el anterior levemente más atenuado, de 80-110 $\mu \mathrm{m}$ de longitud y 15-24 $\mu \mathrm{m}$ de ancho. Cutícula con finas estrías espiraladas. Numerosos cromatóforos discoides y pequeños, sin pirenoides. Paramilon en forma de cuerpos ovales diminutos, distribuidos en toda la célula.
Ecología. - Cosmopolita (Tell y Conforti, 1986). En el bentos (Margalef, 1983).

Distribución geográfica.- En Argentina: Buenos Aires, Córdoba, Corrientes (Tell, 1985). En el Noroeste Argentino: Jujuy (Mirande y Tracanna, 2007); Tucumán (Tracanna et al., 1999).

\section{Euglena proxima Dangeard, Botaniste 8, p. 154, 1902.}

(Fig. 4J)

Células fusiformes a largamente lanceoladas, extremos anterior truncado y posterior adelgazado y terminado en un apéndice caudal, de 40-41 $\mu \mathrm{m}$ de longitud (total) y 12-14 $\mu \mathrm{m}$ de ancho. Cutícula finamente estriada. Numerosos cromatóforos pequeños, lenticulares, sin pirenoides, de posición parietal, generalmente apartados de la región anterior de la célula. Paramilon numerosos, ovoides, que pueden tener o no una depresión central. Apéndice caudal bien diferenciado.

Ecología.- Cosmopolita. En charcos, lagunas, madrajones (Tell y Conforti, 1986).

Distribución geográfica.- En Argentina: Buenos Aires, Córdoba (Tell, 1985). En el Noroeste Argentino: Jujuy (Mirande y Tracanna, 2007); Salta (Salusso, 1998); Tucumán (Tracanna et al., 1994; Mirande y Tracanna, 1995, 2004; Tracanna et al., 1996, 1999).

\section{Lepocinclis Perty, 1852}

Lepocinclis ovum var. bütschlii (Lemm.) Conrad, Mém. Mus. R. Hist. Nat. Bel., 50, ser. 2 (1), p. 39, 1935.

(Fig. 4K)

Células ovoides, extremos anterior redondeado o ligeramente deprimido en el centro y posterior con un ensanchamiento entre el apéndice caudal y dicho borde, de 35-47 $\mu \mathrm{m}$ de longitud (total) y $22-24 \mu \mathrm{m}$ de ancho. Cutícula con estriación levógira. Cromatóforos discoidales y numerosos. Dos paramilon en forma de anillos opuestos. Apéndice caudal de 10-14 $\mu \mathrm{m}$ de largo.

Ecología. - Cosmopolita (Tell y Conforti, 1986). 
Distribución geográfica. - En Argentina: Santa Fe (Tell, 1985). En el Noroeste Argentino: Jujuy (Mirande y Tracanna, 2007).

Trachelomonas Ehrenberg, 1833

Trachelomonas intermedia Dangeard, Botaniste 8, p. 231, 1902.

(Fig. 4L)

Lóriga subhemisférica o elipsoidal, de 20$23 \mu \mathrm{m}$ de longitud y 19-20 $\mu \mathrm{m}$ de ancho. Poro con espesamiento anular, de 2,5-3 $\mu \mathrm{m}$ de diámetro. Cápsula pardo-amarillenta de pared delgada y finamente punteada.

Ecología. - Cosmopolita (Tell y Conforti, 1986).

Distribución geográfica.- En Argentina: Buenos Aires, Corrientes (Tell y Conforti, 1986). En el Noroeste Argentino: Catamarca y Tucumán (Tracanna, 1985); Jujuy (Mirande y Tracanna, 2007).

\section{DISCUSIÓN Y CONCLUSIONES}

De los veintiocho taxones reconocidos en este estudio, diecinueve fueron registrados en dos o menos de los sitios seleccionados, es decir, tuvieron una frecuencia menor de $10 \%$. Aparte del predominio de especies raras, la mayoría fueron cosmopolitas y de medios salinos de acuerdo a las bibliografías consultadas (Krieger, 1937; Huber-Pestalozzi, 1961, 1983; Ramanathan, 1964) y a nuestros resultados. Por otro lado, cabe destacarse que hubieron organismos derivados de los sedimentos y de otros sustratos, lo cual contribuye a la supervivencia de las comunidades algales fluviales (Billen et al., 1994; Izaguirre y Vinocur, 1994; Reynolds et al., 1994). Se puede inferir que la turbulencia ocasionada por brisas y vientos de diferentes intensidades, factor constante y normal en estas regiones, contribuyó a la deriva de especies del bentos al plancton, la cual fue acentuada por la poca profundidad que presentan estos humedales. Esto último, pudo observarse en varias de estas lagunas someras, donde las patas de los flamencos a veces hasta sus rodillas estaban sumergidas, por más que no se dispone de registros numéricos. En conjunción con ello, la importancia de la turbulencia a nivel de la supervivencia de la biota acuática fue destacada por diversos autores (Ambühl, 1960; Margalef, 1983).

En relación a los grupos analizados, el predominio de especies sensibles a la salinidad fue disímil a lo observado en estos cuerpos de agua al estudiarse las cianobacterias (Mirande y Tracanna, 2015). Las altas alcalinidades de las aguas serían indicativas del predominio de bicarbonato, que según Cairns et al. (1972) es asimilado por la mayoría de las algas dulceacuícolas. Esto ocasiona generalmente un aporte de cationes al entorno, entre los cuales sodio y potasio son los más comunes. Diversos estudios experimentales han demostrado que ambos iones son nutrientes esenciales para el crecimiento de las azules (Provasoli, 1969; Cole, 1988) y, por ende, las condiciones de estos ambientes extremos las favorecerían. Caziani y Derlindati (1999) indicaron para el complejo Vilama concentraciones entre 60$13800 \mathrm{mg} / \mathrm{l}$ de sodio para el período húmedo (enero/97) y 178-48000 para el período seco (octubre/97), por lo cual no sería un factor limitante. Lo comentado daría indicios de que tanto las algas verdes (53\% de los taxones observados) como los euglénidos (7\%) manifestaron una sensibilidad salina, por lo cual sería de interés conocer sus tolerancias por ejemplo hacia estos cationes.

Se contribuyó al conocimiento de la ficoflora de los ambientes leníticos seleccionados, siendo los taxones de clorofitas y euglenofitas enunciados en esta publicación nuevas citas para estos humedales. Por otro lado, Ulothrix pseudoflacca var. salina se menciona por primera vez para el Noroeste Argentino, habiendo sido citada en Argentina la especie para laguna Mar Chiquita, en la provincia de Córdoba (Bucher y Abril, 2006).

Por último, es fundamental destacar la fragilidad de estos ecosistemas más aún con la intervención humana, especialmente la minería a cielo abierto, la cual no sólo conduce a la extracción de minerales y contaminaciones consiguientes sino que las perforaciones para el suministro de agua están 
ocasionando el secado de vegas o bofedales, imprescindibles para la supervivencia de diferentes especies, además de ser reservorios de agua dulce.

\section{AGRADECIMIENTOS}

A la Dra. Sandra Caziani y su equipo de trabajo por hacernos conocer en aquel verano de 2005 tan bellos lugares, además de tratarse de un grupo humano excepcional. También queremos agradecer a la Lic. Inés Jaume del Instituto de Iconografía de la Fundación Miguel Lillo por el pasado en tinta de los dibujos.

\section{BIBLIOGRAFÍA}

Ambühl H. 1960. Die Bedeutung der Strömung als ökologister Faktor. Schweizerische zeitschrift fur Hydrologie 21: 133264.

Billen G., Garnier J., Hanset P. 1994. Modelling phytoplankton development in whole drainage networks: the RIVERSTRAHLER Model applied to the Seine River System. En: Descy J. -P., Reynolds C. S., Padisák J. (editors) Phytoplankton in Turbid Environments: Rivers and Shallow Lakes. Hidrobiologia 294: 119-137.

Bourrelly P. 1972. Les algues d'eau douce. I: Les algues vertes. Editions Nérée Boubée et Cie. Paris. 572 pp.

Bourrelly P. 1985. Les algues d'eau douce. Tome III. Les Algues Bleues et Rouges. Les Eugléniens, Peridiens et Cryptomonadines. Editions Nérée Boubée et Cie. Paris. 606 pp.

Bucher E. H., Abril A. B. 2006. Limnología Biológica. En: Bucher E. H. (editor). Bañados del río Dulce y Laguna Mar Chiquita [Córdoba, Argentina]. Academia Nacional de Ciencias (Córdoba, Argentina): 117-137.

Cairns J. [Jr.], Lanza G. R., Parker B. C. 1972. Pollution related structural and functional changes in aquatic communities with emphasis on freshwater algae and protozoa. Proceeding of the Academy of Natural Sciences of Philadelphia 124 (5): 79-127.

Canevari P., Blanco D. E., Bucher E. H., Castro G., Davidson I. 1998. Los humedales de la Argentina. Wetlands International, Publ. 46: 1-208.

Caziani S. M., Derlindati E. J. 1999. Humedales altoandinos del Noroeste de
Argentina: su contribución a la biodiversidad regional. En: Malvárez, A. I. (editor). Tópicos sobre humedales subtropicales y templados de Sudamérica. Oficina Regional de Ciencia y Tecnología de la UNESCO para América Latina y el Caribe -ORCYT - Montevideo, Uruguay: 1-13.

Caziani S. M., Derlindati E. J. 2000. Abundance and habitat of High Andean flamingos in Northwestern Argentina. Waterbirds 23 (Special Publication 1): 121-133.

Caziani S. M., Derlindati E. J., Tálamo A., Sureda A. L., Trucco C. E., Nicolossi G. 2001. Waterbird Richness in Altiplano Wetlands of Northwestern Argentina. Waterbirds 24 (1): 103-117.

Coconier E. 2005. Reporte final aves acuáticas en la Argentina. En: Blanco D. E. (editor). La Conservación de las Aves Acuáticas para las Américas (Waterbird Conservation for the Americas]. Wetlands International. Buenos Aires: 2-137.

Cole G. A. 1988. Manual de Limnología. Editorial Hemisferio Sur S.A. (primera edición). Buenos Aires. 405 pp.

Comas González A. 1996. Las Chlorococcales dulciacuícolas de Cuba. J. Cramer Ed. Berlin. Stuttgart. Biblioteca Phycologica. 192 pp.

Del Giorgio P. 1988. Nuevos taxa de algas de agua dulce para la República Argentina. Boletín de la Sociedad Argentina de Botánica 25 (3-4): 563-573.

Di Giacomo A. S., Coconier E. 2005. Conservación de aves en Catamarca. En: Di Giacomo A. S. (editor). Áreas importantes para la conservación de las aves en Argentina. Sitios prioritarios para la conservación de la biodiversidad. Temas de Naturaleza y Conservación 5. Aves Argentinas/Asociación Ornitológica del Plata. Buenos Aires, Argentina: 69-71.

Dirección de Recursos Ictícolas y Acuícolas. 2006. Secretaría de Ambiente y Desarrollo Sustentable. Definiciones y conceptos sobre humedales. Programa Panamericano de Defensa y Desarrollo de la diversidad biológica, cultural y social, asociación civil (ProDiversitas). Buenos Aires (Argentina). Internet: www. bioetica.org.

Fjeldsa J. 1985. Origen, evolution and status of the avifauna of the Andean Wetlands. En: Buckley P. A. (editor). Neotropical Ornithology. Washington D. C. American Ornithologist's Union. Ornithological Monographs 36: 85-112. 
Halloy S. 1978. Contribución al estudio de la zona de Huaca Huasi, Cumbres Calchaquíes (Tucumán, Argentina). La biota actual en relación con la geología histórica. Facultad de Ciencias Naturales e Instituto Miguel Lillo, Universidad Nacional de Tucumán. Tucumán, Argentina. 50 pp.

Halloy S. 1982. Contribución al estudio de la zona de Huaca Huasi, Cumbres Calchaquies (Tucumán, Argentina). Climatología y edafología en relación con la composición y adaptación de las comunidades bióticas. Tesis Doctoral. Facultad de Ciencias Naturales e Instituto Miguel Lillo, Universidad Nacional de Tucumán. Tucumán, Argentina. 826 pp.

Huber-Pestalozzi G. 1961. Das Phytoplankton des Süsswassers, Chlorophyceae. En: Thienemann A. (editor). Die Binnengewasser, 16 (5), VI-XII, E. Schweizerbart'sche Verlagsbuchhandlung, Stuttgart. $744 \mathrm{pp}$.

Huber-Pestalozzi G. 1983. Das Phytoplankton des Süsswassers, Chlorophyceae. Ordnung Chlorococcales. En: Thienemann A. (editor) Die Binnengewasser, 16 (7), E. Schweizerbart'sche Verlagsbuchhandlung, Stuttgart. 1044 pp.

Izaguirre I., Vinocur A. 1994. Algal assemblages from lakes of the Salado River Basin (Argentina). En: Descy J. -P., Reynolds C. S., Padisák J. (editors) Phytoplankton in Turbid Environments: Rivers and Shallow Lakes. Hidrobiologia 294: 57-64.

INTA-UNsa. 2009. Adecuación a un SIG del estudio "Los Suelos del NOA [Salta y Jujuy]». El Medio Natural. Salta, Argentina. $14 \mathrm{pp}$.

Kammerer G. 1938. Volvocalen und Protococcalen aus dem unteren Amazonas-gebiet. Sitzungsberichte der mathem.-naturw. KI., Abt. I, 147. Bd., 5. bis 10. Heft.: 183-228.

Klochkova T. A., Cho G. Y., Boo S. M., Chung K. W., Kim S. J., Kim G. H. 2008. Interactions between marine facultative epiphyte Chlamydomonas sp. (Chlamydomonadales, Chlorophyta) and ceramiaceaen algae (Rhodophyta). Journal of Environmental Biology: 427-435.

Krieger W. 1937. Die Desmidiaceen Europas mit Berücksichtigung der ausserenuropäischen Arten, I Rabenhorst's, Kriptogamen-Flora von Deustschland, österreich und der Schweiz, 13 (1): 1-712.

Lacoste de Díaz E. N. 1979. Algas de aguas continentales en la Argentina III. Desmidiaceae de Misiones: Closteriaceae. Lilloa 35 (2): 47-63.
Locascio de Mitrovich C. 1986. Presencia de Pseudoboeckella palustris Harding (Crustacea - Copepoda) en lagunas de altura del Noroeste Argentino. Neotropica 32 (87): 13-21.

Locascio de Mitrovich C., Ceraolo M. 1999. Copépodos de algunos cuerpos de agua en los departamentos de Belén y Antofagasta de la Sierra (Catamarca - Argentina). Resúmenes IV Taller sobre Cangrejos y Cangrejales y I Jornadas Argentinas de Carcinología, Buenos Aires. $44 \mathrm{p}$.

Locascio de Mitrovich C., Villagra de Gamundi A., Juárez J., Ceraolo M. 2005. Características limnológicas y zooplancton de cinco lagunas de la Puna - Argentina. Ecología en Bolivia 40 (1): 10-24.

Maidana N. I., Seeligmann C. 2006. Diatomeas (Bacillariophyceae) de ambientes acuáticos de altura de la provincia de Catamarca, Argentina II. Boletín Sociedad Argentina Botánica 41: 1-13.

Margalef R. 1983. Limnología. Ed. Omega, S.A. $1010 \mathrm{pp}$.

Martínez De Marco S. N. 1995. Algas edáficas de Tucumán (Argentina). Lilloa 38 [2]: 5-39.

Mirande V., Tracanna B. 1995. Estudio cualitativo del fitoplancton del embalse Río Hondo (Argentina): I. Criptogamie, Algologie 16 (4): 211-232.

Mirande V., Tracanna B. 2004. Fitoplancton del río Gastona (Tucumán, Argentina). Cyanophyta, Chlorophyta, Euglenophyta y Rhodophyta. Iheringia, Série Botânica 59 (1): 35-58.

Mirande V., Tracanna B. 2007. Diversidad de cianobacterias, clorofitas y euglenofitas en humedales de altura (Jujuy, Argentina). Lilloa 44 (1-2): 39-59.

Mirande V., Tracanna B. 2009. Estructura y controles del fitoplancton en humedales de altura. Ecología Austral 19: 119-128.

Mirande V., Tracanna B. 2015. Cianobacterias de humedales de altura del Noroeste Argentino. Lilloa 52 (2): 122-141.

Moraña L. B. 1998. Estudio de la calidad del agua en un subsistema de ríos de la provincia de Salta sometida a acción antrópica. Tesis Magíster. Universidad Nacional del Litoral. 88 pp.

Morlans M. C. 1995. Regiones Naturales de Catamarca. Provincias Geológicas y Provincias Fitogeográficas. Editorial Científica Universitaria de la Universidad Nacional de Catamarca. Revista de Ciencia y Técnica II (2): 1-42.

Paggi J. C., Villagra de Gamundi A. 1980. Sobre la presencia de Pleuroxus caca 
Harding (Crustacea - Cladocera) en cuerpos de agua de alta montaña de la provincia de Tucumán, Argentina. Acta Zoológica Lilloana 36 (1): 131-138.

Parra Barrientos 0. 0. 1979. Revision der Gattung Pediastrum Meyen (Chlorophyta). J. Cramer Ed. Berlin, Stuttgart. Bibliotheca Phycologica 48: 1-183.

Provasoli L. 1969. Algal nutrition and eutrophication. En: Eutrophication: causes, consequences, correctives. National Academy of Sciences. Washington, D. C. : 574-593.

Ramanathan K. R. 1964. Ulothricales. Indian Council of Agricultural Research. Educational Board. New Delhi. 188 pp.

Reynolds C. S., Descy J. P., Padisák J. 1994. Are phytoplankton dynamics in rivers so different from those in shallow lakes? Hidrobiologia 294: 1-7.

Salusso M. M. 1998. Evaluación de la calidad del agua de dos ríos del valle de Lerma [Salta] sometidos a acción antrópica. Tesis Magíster. Universidad Nacional del Litoral. $84 \mathrm{pp}$.

Seeligmann C. T. 1998. Evaluación de la estructura y dinámica ficológica en el río Salí (Tucumán - Argentina), en relación al impacto de la contaminación antropogénica. Tesis Doctoral. Facultad de Ciencias Naturales e Instituto Miguel Lillo. Universidad Nacional de Tucumán. $191 \mathrm{pp}$

Seeligmann C., Maidana N. I. 2003. Diatomeas de la provincia de Catamarca (Argentina). Boletín de la Sociedad Argentina Botánica 38: 39-50.

Seeligmann C. T., Tracanna B. 1994. Limnología del embalse El Cadillal (Tucumán, Argentina) II: estudio cualitativo del fitoplancton. Cryptogamie, Algologie 15 (1): 19-35.

Seeligmann C., Tracanna B. C., Martínez De Marco S., Isasmendi S. 2001. Algas fitoplanctónicas en la evaluación de la calidad del agua de sistemas lóticos en el Noroeste Argentino. Limnetica 20 (1): 123-133.

Seeligmann C., Maidana N. I., Morales M. 2008. Diatomeas (Bacillariophyceae) de humedales de altura de la provincia de Jujuy-Argentina. Boletín de la Sociedad Argentina Botánica 43 (1-2): 1-17.

Sureda A. L., Caziani S. 2007 a. Laguna Grande. En: Di Giacomo A. S., De Francesco M. V., Coconier E. G. (editores). Áreas importantes para la conservación de las aves en Argentina. Sitios prioritarios para la conservación de la biodiversidad. Temas de Naturaleza y
Conservación 5. CD-ROM. Edición Revisada y Corregida. Aves Argentinas / Asociación Ornitológica del Plata, Buenos Aires: 79.

Sureda A. L, Caziani S. 2007 b. Laguna La Alumbrera. En: Di Giacomo A. S., De Francesco M. V., Coconier E. G. (editores]. Áreas importantes para la conservación de las aves en Argentina. Sitios prioritarios para la conservación de la biodiversidad. Temas de Naturaleza y Conservación 5. CD-ROM. Edición Revisada y Corregida. Aves Argentinas / Asociación Ornitológica del Plata, Buenos Aires: 80-81.

Sureda A. L., Caziani S. 2007 c. Laguna Purulla. En: Di Giacomo A. S., De Francesco M. V., Coconier E. G. (editores). Áreas importantes para la conservación de las aves en Argentina. Sitios prioritarios para la conservación de la biodiversidad. Temas de Naturaleza y Conservación 5. CD-ROM. Edición Revisada y Corregida. Aves Argentinas / Asociación Ornitológica del Plata, Buenos Aires: 81.

Sureda A. L., Caziani S., Moschione F. 2005. Reserva Provincial y de la Biosfera Laguna Blanca. En: Di Giacomo A. S. (editor]. Áreas importantes para la conservación de las aves en Argentina. Sitios prioritarios para la conservación de la biodiversidad. Temas de Naturaleza y Conservación 5. Aves argentinas / Asociación Ornitológica del Plata. Buenos Aires: 73-74.

Tell G. 1985. Catálogo de las Algas de Agua Dulce de la República Argentina. Bibliotheca Phycologica, 70. Ed. J. Cramer. Alemania. $283 \mathrm{pp}$.

Tell G., Conforti, V. T. D. 1986. Euglenophyta pigmentadas de la Argentina. Facultad de Ciencias Naturales de la UBA. Buenos Aires. 301 pp.

Tell G., Izaguirre I., O’Farrell I. 1994. Ecological and Taxonomical remarks on the Desmid Flora of the Lower Uruguay River Basin (Argentina). Ed. J. Cramer. Berlin, Stuttgart. Bibliotheca Phycologica 96: 1-141.

Tracanna B. C. 1981. Estudio taxonómico de las Chlorophyta de los alrededores de Tucumán (incluidas algunas consideraciones ecológicas]. Ópera Lilloana 32 : $1-91$.

Tracanna B. C. 1985. Algas del Noroeste Argentino (excluyendo a las Diatomophyceae). Ópera Lilloana 35: 1-136.

Tracanna B. C., Martínez De Marco S. N. 1997. Ficoflora del río Salí y sus tribu- 
tarios en áreas del embalse Dr. C. Gelsi [Tucumán, Argentina]. Natura Neotropicalis 28 (1): 23-38.

Tracanna B. C., Mirande V., Seeligmann C. 1994. Variaciones del fitoplancton superficial del embalse Río Hondo (Tucumán - Santiago del Estero, Argentina), en relación a la actividad azucarera. Tankay 1: 80-82.

Tracanna B. C., Seeligmann C., Mirande V. 1996. Estudio comparativo de dos embalses del Noroeste Argentino. Revista Asociación Ciencias Naturales Litoral 27 (1): 13-22.

Tracanna B. C., Seeligmann C. T., Mirande V., de Parra L. B., de Plaza M. T., Molinari F. M. 1999. Cambios espaciales y temporales del fitoplancton en el embalse Río Hondo (Argentina). Boletín de la Sociedad Argentina Botánica 34 (12): 101-105.

Uherkovich G. 1966. Die Scenedesmus-Arten Ungarns Verlag der Ungarischen Akad- emie der Wissenschaften Akadémiai Kiadó. Budapest. 173 pp.

Van Den Hoek C., Mann D. G., Jahns H. M. 1995. Algae. An Introduction to Phycology. 3o edition. London. Cambridge University Press. $623 \mathrm{pp}$.

Vecinos del Humedal. 2013. Argentina: Humedales Sitios Ramsar «Humedales Península Valdés». http://vecinosdelhumedal. blogspot.com.

Villagra de Gamundi A. 1994. Aspectos bioecológicos de ambientes lénticos de alta montaña 4000 m snm -Tucumán- Argentina) con especial referencia al zooplancton. Tankay 1: 116-119.

Villagra de Gamundi A. 1998. Tipificación de ambientes acuáticos leníticos de la provincia de Tucumán en base a los atributos del zooplancton y algunas características limnológicas. Tesis Doctoral, Facultad de Ciencias Naturales e Instituto Miguel Lillo, Universidad Nacional de Tucumán. Tucumán, Argentina. 465 p. 